\title{
Imaging Features of Neurocysticercosis: Analysis of 57 Consecutive Patients
}

\author{
Anqi XIAO' ${ }^{1}$, Hanjiang ZENG ${ }^{2}$, Jiahe $\mathrm{XIAO}^{2}$, Xiaoke ZHANG ${ }^{3}$, Chao YOU ${ }^{1}$ \\ 'Sichuan University, West China Hospital, Department of Neurosurgery, Chengdu, China \\ ${ }^{2}$ Sichuan University, West China Hospital, Department of Radiology, Chengdu, China \\ ${ }^{3}$ Sichuan University, West China Hospital, Department of Infectious Diseases, Chengdu, China
}

\section{ABSTRACT}

AIM: To investigate the imaging features of neurocysticercosis and to provide valuable information about the diagnosis and treatment for clinicians.

MATERIAL and METHODS: The data of 71 consecutive cases of neurocysticercosis, diagnosed by computed tomography (CT) and magnetic resonance imaging (MRI) between January 2009 and January 2014 in our institute were collected. Of all the cases, 57 patients met the recognized diagnostic criteria of neurocysticercosis. In this series, enzyme-linked immunosorbent assay (ELISA) examination and Cysticercosis IgG were positive in 55 cases and 3 cases, respectively. Among them, neurocysticercosis was confirmed surgically in 11 cases. Seven cases had CT examinations, including enhanced CT in one case. Fifty-four cases had MRI examinations, including contrast-enhanced MRI in 46 cases. Therapeutically, 32 patients received albendazole, while 25 patients received praziquantel. As a surgical treatment, 9 patients underwent lesionectomy, and 1 patient underwent lesionectomy plus shunt insertion for hydrocephalus.

RESULTS: Parenchymal cysticerci in 53 cases (92.9\%), subarachnoidal cysticerci in 39 cases (68.4\%), ventricular cysticerci in 13 cases $(22.8 \%)$ and spinal cysticerci in 1 case were demonstrated on CT and MRI scans. In total, the intracranial cysticerci involving multiple locations simultaneously were found in 39 patients. Thirty-five cases had associated leptomeningitis, 10 cases had hydrocephalus. In 1 case, the cysticerci were located outside of the central nervous system.

CONCLUSION: The imaging findings of the cysticerci, including their location, numbers, cystic sizes, capsular thickness, densities and signals of the scolexes, as well as the peripheral edema, have distinct value in making possible timely diagnosis of neurocysticercosis for clinicians.

KEYWORDS: Central nervous system, Computed tomography, Cysticercosis, Diagnosis, Magnetic resonance imaging

\section{INTRODUCTION}

$\mathrm{C}$ ysticercosis is a kind of parasitic disease caused by larva of Taenia solium, with predilection sites of muscles, eyes and central nervous system (CNS). Apart from parasitizing pigs, it could also infect the human body.

Generally, cysticercosis is prevalent in developing countries of Asia, Africa, or Latin America (except Islamic countries). However, in recent years, the incidence of cysticercosis in developed countries is rising due to immigration $(2,10)$. The symptoms and imaging features of neurocysticercosis vary with its different infectious phases, and that makes it easier to misdiagnose it as other diseases.

The aim of this article is to explore imaging characteristics of cysticercosis in CNS by retrospectively analyzing imaging data of neurocysticercosis at our institute and to provide valuable information for the clinicians in making diagnosis and choosing proper medical or surgical treatment. 


\section{MATERIAL and METHODS}

\section{Patient Population}

Through picture archiving and communication system (PACS) and Hospital information system (HIS) by searching the keyword 'neurocysticercosis', the data of 71 consecutive cases of neurocysticercosis diagnosed by computed tomography (CT) and magnetic resonance imaging (MRI) between January 2009 and January 2014 in our institute were collected. Of all, 57 patients, including 33 males and 24 females, the age range was 11-71 years with a mean age of 37.3 years, confirmed with diagnostic criteria of neurocysticercosis according to the diagnostic standards formulated by a group of experts in 2001 (4).

\section{Examinations}

The clinical symptoms included headache in 38 patients, seizures in 18 patients, movement disorders in 12 patients and consciousness disorders in 7 patients. In this series, 55 cases were positive on enzyme-linked immunosorbent assay (ELISA) examination, 3 cases were positive for cysticercosis IgG; 11 cases were confirmed by surgery (including 1 case of biopsy). Seven cases had CT examinations, including enhanced CT in 1 case, using Ultravist $370(1 \mathrm{ml} / \mathrm{kg})$ as contrast-enhanced agent. Fifty-four cases had MRI examinations, including sequences of T1-weighted imaging (T1WI), T2-weighted imaging (T2WI), and fluid attenuated inversion recovery (FLAIR), acquiring axial, coronal and sagittal data. Among them, 46 cases underwent contrast-enhanced MRI simultaneously by injecting gadopentetate dimeglumine $(0.1 \mathrm{mmol} / \mathrm{kg})$. CT devices included Sensation 16 Row and Somatom Definition AS+ (Siemens company), and Brilliance 16 row (Philips company). MR devices included Trio Tim 3.0T, Avanto 1.5T and Sonata 1.5T (Siemens company), SIGNA EXCITE 3.0T (GE company), Achieva 3.0T (Philips company), and MRT200SP5 1.5T (Toshiba company).

\section{Treatment}

As medical therapy, 32 patients received albendazole, while 25 patients received praziquantel. As a surgical treatment, 9 patients underwent lesionectomy, and 1 patient underwent lesionectomy plus shunt insertion for hydrocephalus.

\section{RESULTS}

According to the location, neurocysticercosis is classified into parenchymal cysticerci, subarachnoidal cysticerci, ventricular cysticerci and spinal cysticerci (Table I).

\section{Parenchymal Cysticerci}

Parenchymal cysticerci were found in 53 cases (92.9\%), including multiple lesions in 46 patients (86.8\%). Based on the imaging, cerebral subcortical cysticerci in 51 patients, basal ganglion cysticerci in 21 patients and posterior fossa cysticerci in 15 patients were analyzed. In this series, 3 cases were non-cystic calcified nodules (Figure 1A,B), while 50 cases had cysts and scolexes on MRI and CT. In cystic lesions, the sizes were inconsistent. Cysts over $15 \mathrm{~mm}$ in diameter, ranging from 15 to $40 \mathrm{~mm}$ with a mean diameter of
$20 \mathrm{~mm}$, were found in 8 cases. On MRI, these lesions were with thin capsules with isointensity on $\mathrm{T} 1 \mathrm{WI}$ and hypointensity on T2WI, and apparent scolexes with signals equal to cerebral parenchyma. On contrast-enhanced T1WI, the capsules and scolexes enhanced slightly. In addition, mild perifocal edema was displayed on T2WI and FLAIR in 4 patients (Figure 2A-C). Cysts smaller than $15 \mathrm{~mm}$, with a mean diameter of $8 \mathrm{~mm}$, were found in 51 patients. Their scolexes and thicker capsules were shown as hypointensity on T2WI and apparent enhancement on gadolinium-enhanced T1WI. On T2WI and FLAIR, 27 cases had serious perifocal edema (Figure $3 A-C$ ). Meanwhile, lesions about $5 \mathrm{~mm}$ in mean diameter with mild perifocal edema were noticed in 30 patients (Figure 4A-D). In 3 cases examined by $\mathrm{CT}$, the scolexes appeared as hyperdensities suggesting calcifications. On T2WI, a typical target-like pattern formed by high signal cystic fluid with low signal scolex could be seen (Figure 5A-D). In 1 case, multiple parenchymal lesions without perifocal edema were displayed as peripheral hypointensities on both T1WI and T2WI, but central patchy hyperintensities on T2WI, just like cavernous hemangioma. In parenchymal

Table I: The Imaging Findings of the Involved Locations and Complications in 57 Patients

\begin{tabular}{|c|c|c|}
\hline & $\mathbf{n}$ & $\%$ \\
\hline \multicolumn{3}{|l|}{ Location of involvement } \\
\hline Brain parenchyma & 53 & $93 \%(53 / 57)$ \\
\hline Subcortex & 51 & $96 \%(51 / 53)$ \\
\hline Basal ganglion & 21 & $40 \%(21 / 53)$ \\
\hline Cerebellum & 11 & $21 \%(11 / 53)$ \\
\hline Brain stem & 4 & $8 \%(4 / 53)$ \\
\hline Subarachnoid space & 39 & $68 \%(39 / 57)$ \\
\hline Cerebral convexity & 34 & $87 \%(34 / 39)$ \\
\hline Suprasellar cistern & 4 & $10 \%(4 / 39)$ \\
\hline $\begin{array}{l}\text { Ambient-quadrigeminal } \\
\text { cistern }\end{array}$ & 4 & $10 \%(4 / 39)$ \\
\hline Cerebellopontine Angle & 2 & $5 \%(2 / 39)$ \\
\hline Ventricles & 13 & $23 \%(13 / 57)$ \\
\hline Lateral ventricle & 11 & $85 \%(11 / 13)$ \\
\hline The $4^{\text {th }}$ ventricle & 2 & $15 \%(2 / 13)$ \\
\hline Spine & 1 & $2 \%(1 / 57)$ \\
\hline Cervical section & 1 & $100 \%(1 / 1)$ \\
\hline Extra central nervous system & 1 & $2 \%(1 / 57)$ \\
\hline \multicolumn{3}{|l|}{ Complication } \\
\hline Hydrocephalus & 10 & $18 \%(10 / 57)$ \\
\hline Leptomeningitis & 35 & $74 \%\left(35 / 47^{\star}\right)$ \\
\hline
\end{tabular}



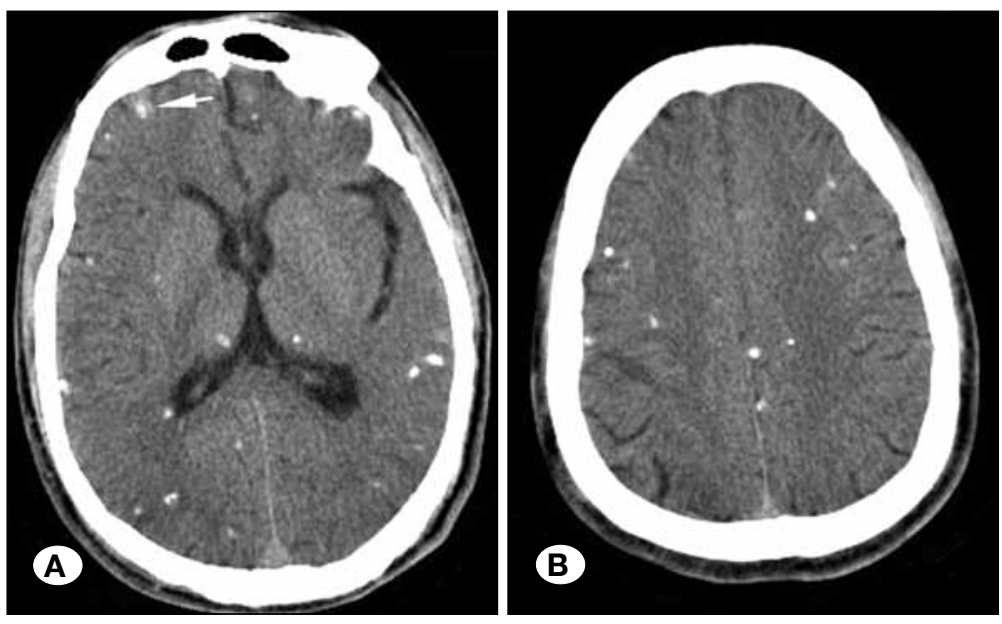

Figure 1: A, B) The plain CT of a 28-year-old male with neurocysticercosis. The sporadic calcified scolexes as punctate nodules with hyperdensities were seen in bilateral parenchyma, without perifocal edema. A calcified scolex within a slightly high density capsule was shown in the right frontal lobe (A; Arrow). These imaging findings were consistent with the cysticerci in calcified-nodular stage.
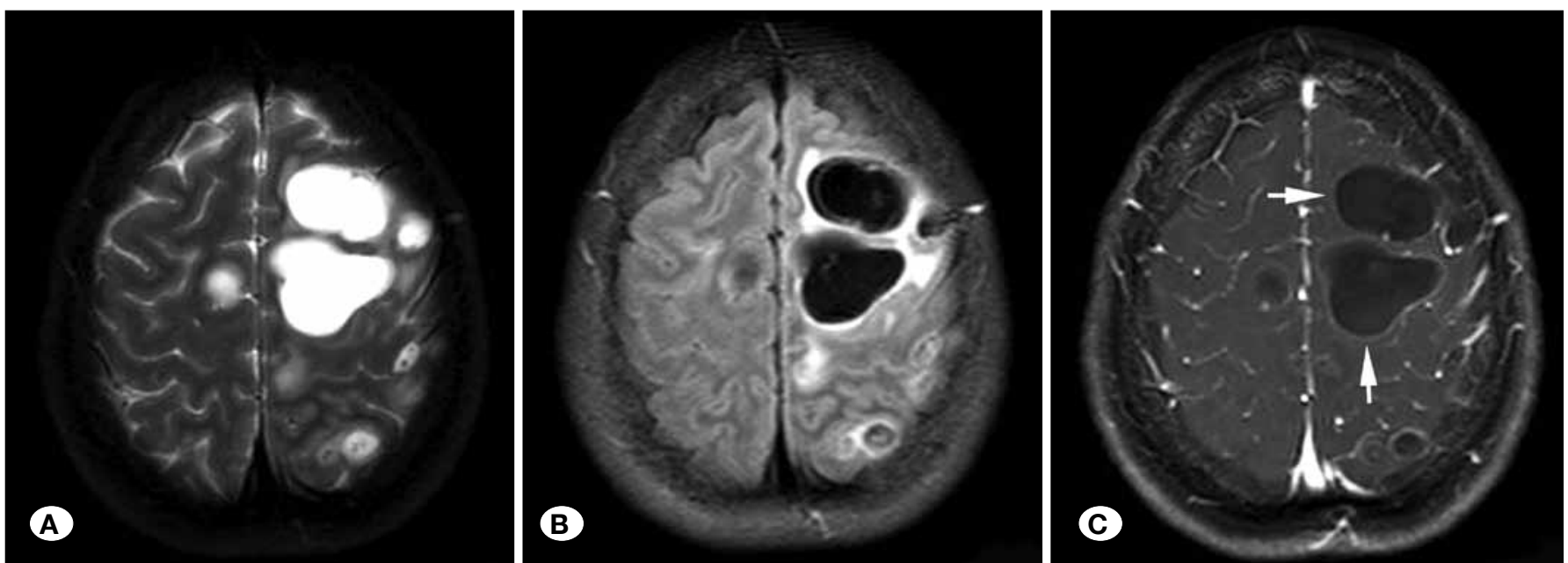

Figure 2: The MR imaging of a 35-year-old female with neurocysticercosis. A) T2WI, B) FLAIR, C) Contrast-enhanced T1WI. Multiple cysticerci with various sizes were seen in bilateral cerebral hemispheres. The largest two cysts in the left frontal lobe in the vesicular stage were seen with mild perifocal edema and thin capsules with slight enhancement (C. Arrows).
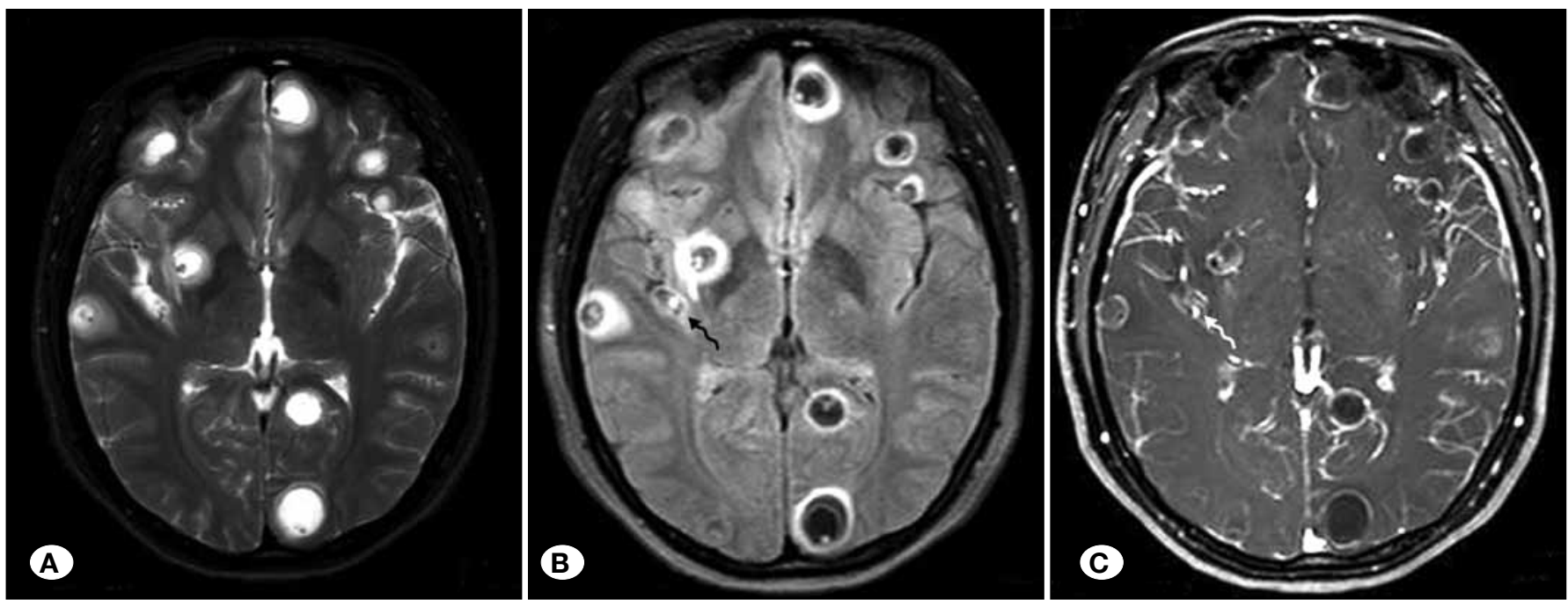

Figure 3: The MR imaging of a 35-year-old female with neurocysticercosis. A) T2WI, B) FLAIR, C) Contrast-enhanced T1WI. Numerous lesions, with diameters of $0.5-1.5 \mathrm{~cm}$, were displayed in bilateral parenchyma. On T2WI, the cystic fluid appeared as hyperintensity, and their scolexes and capsules were shown as relative hypointensities. The pericystic edema as an obvious high signal was seen on T2W and FLAIR. On contrast-enhanced T1WI, these lesions were with apparent ring-like enhancement, corresponding to the manifestations of cysticercosis in colloidal-vesicular stage. A lesion located in the right Sylvian fissure was seen as well (C; Curved Arrow). 
series, the lesions with various sizes and imaging features were found in 19 patients. In superficial cortical lesions, the adjacent sulci were enhanced on contrast-enhanced T1WI in 33 patients.

\section{Subarachnoidal Cysticerci}

Subarachnoidal cysticerci were found in 39 cases (68.4\%) with multiple lesions. The lesions were located in cerebral convexity in 34 patients, suprasellar cistern in 4 cases, quadrigeminal cistern in 4 cases, Sylvian fissure in 3 cases and cerebellopontine angle (CPA) in 2 cases. The cysts of the Sylvian fissures or cisterns were big in size, generally was over $10 \mathrm{~mm}$ in diameter. The biggest one in our series was located in the quadrigeminal cistern and $50 \mathrm{~mm}$ in size. On the imaging, subarachnoidal cysticerci had thin capsules, but rarely visible scolexes, and the cystic enhancement varied from person to person (Figure $6 \mathrm{~A}-\mathrm{C}$ ). In the case of aggregation, the multiple cysts were demonstrated as a botryoid-like shape on imaging.
In 27 cases, the adjacent leptomeninges were enhanced on contrast-enhanced T1WI.

\section{Ventricular Cysticerci}

Ventricular cysticerci were found in 13 cases (22.8\%), including 11 cases with lateral ventricular cysticerci and 2 cases with $4^{\text {th }}$ ventricular cysticerci. The cysts with ependymal attachment with mean diameter of $7 \mathrm{~mm}$ were seen in 11 patients, associated with enhancement in their scolexes, capsules, as well as the adjacent ependymal layers on contrastenhanced MRI. In the other 2 cases, the lesions floated in the ventricular cavities (Figure 7A,B). The sizes of $4^{\text {th }}$ ventricular cysticerci were relatively larger and the diameter reached 40 $\mathrm{mm}$ maximum (Figure 8A-C). Compared with cerebrospinal fluid (CSF) signals, the cysts' signals were relatively higher on T1WI, but lower on T2WI. Meanwhile, the features of thin capsules and invisible scolexes were noticed.
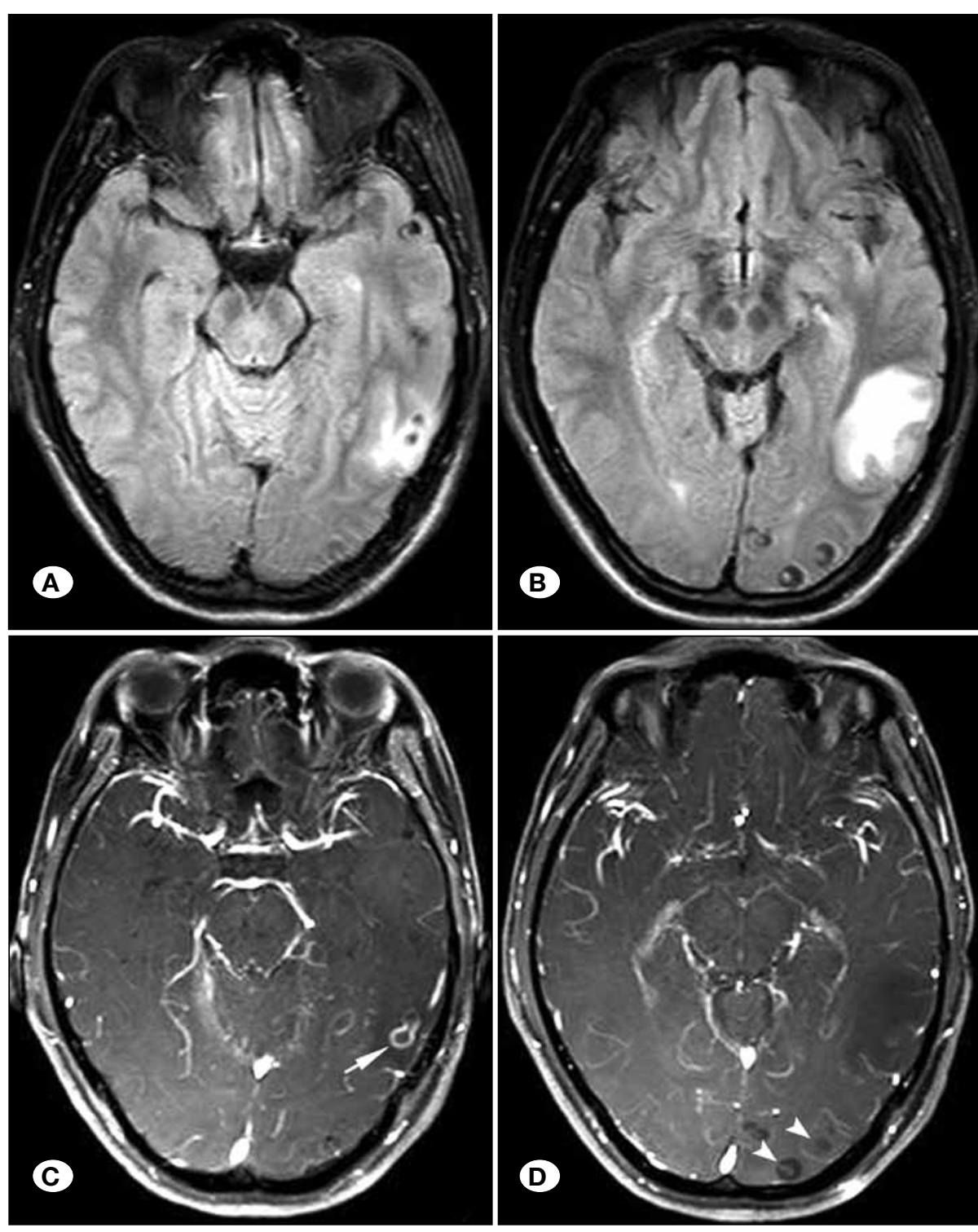

Figure 4: The MR imaging of a 36-yearold male with neurocysticercosis.

A, B) FLAIR; C, D) contrast-enhanced T1WI. Two cysticerci in the left temporal lobe with high signal edema on FLAIR and remarkable ring-like enhancement of capsules on contrast-enhanced T1WI (C; Arrow) were shown, indicating them in colloidal-vesicular stage. By contrast, other two lesions in the ipsilateral parietal lobe were found with little perifocal edema on FLAIR and mild enhancement on contrast-enhanced T1WI (D; Arrowhead), consistent with imaging features of the cysticerci in granular-nodular stage. 

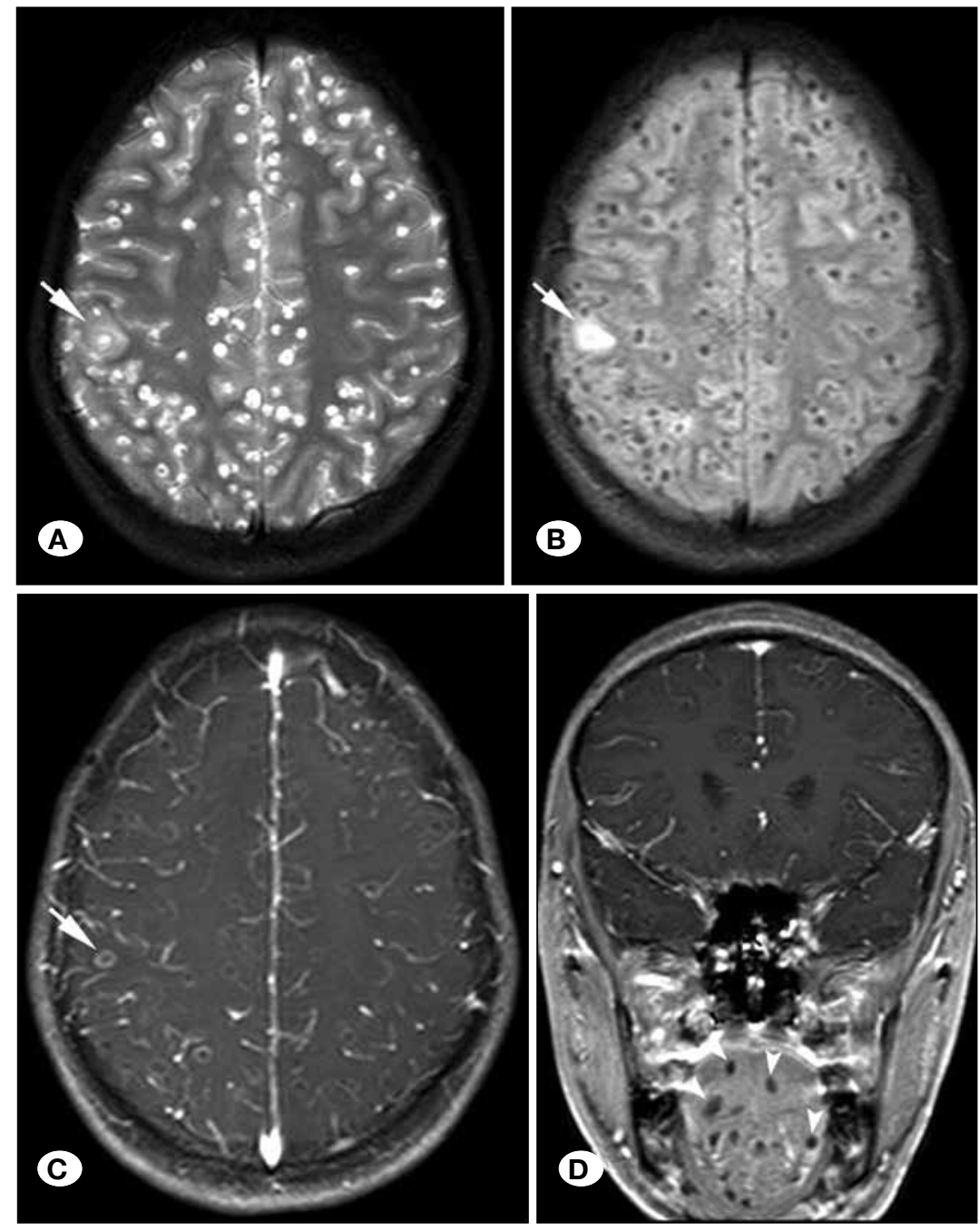

Figure 5: The MR imaging of a 13-year-old girl with neurocysticercosis. A) T2WI, B) FLAIR, C) Axial contrast-enhanced T1WI, D) Coronal contrast-enhanced T1WI. The diffuse cysticerci distributed in bilateral hemispheres, with diameter about $5 \mathrm{~mm}$, were demonstrated. The lesions with low signal scolex and high signal cystic fluid were found on T2WI. Most cysticerci showed little perifocal edema on T2WI and FLAIR, and enhanced cystic capsules on contrastenhanced MRI, indicating the cysticerci in the granular-nodular stage. However, one lesion with obvious edema as hyperintensity on T2WI and FLAIR and remarkable enhanced capsule on contrast-enhanced T1WI was found in the right parietal lobe (A-C; Arrow). These inflammatory manifestations on imaging suggest its colloidalvesicular stage. On coronal enhanced T1WI, besides the cerebral lesions, multiple cysticerci in the patient's lingualis were found as well. (D; Arrowheads).
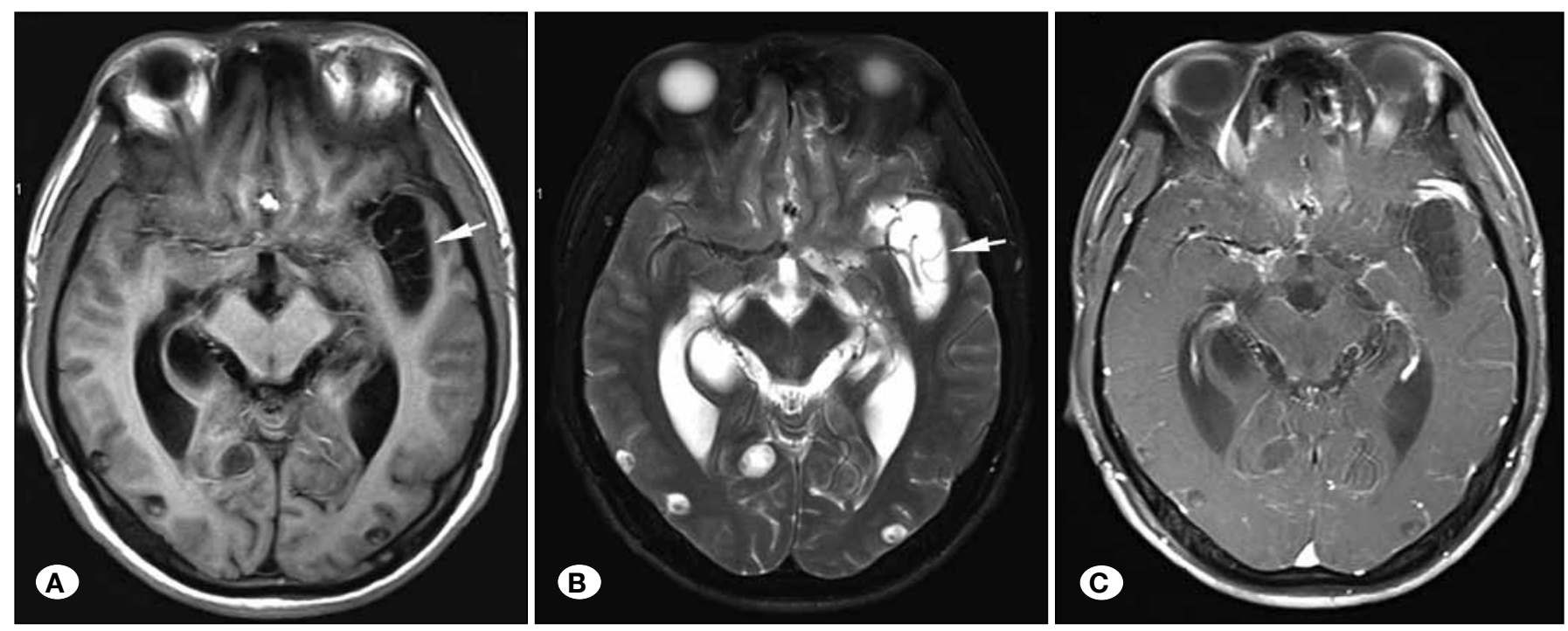

Figure 6: The MR imaging of a 51-year-old male with neurocysticercosis. A) T1WI, B) T2WI, C) Contrast-enhanced T1WI. The several cysticerci with thin capsular walls located in dilated left Sylvian fissure were found (A, B; Arrow). The contrast-enhanced T1WI demonstrated that the capsules of the lesions had no obvious enhancement. In bilateral cerebral parenchyma, multiple cysticerci were found simultaneously. 


\section{Spinal cysticerci}

Spinal cysticerci were found only in 1 case, located at the C1-2 level, and about $8 \mathrm{~mm}$ in diameter. On MRI, the lesions appeared as peripheral hypointensity on T1WI and T2WI, and central hyperintensity on T2WI, without perifocal edema (Figure 9).

In our series, the cysticerci involving multi-sites mentioned above were revealed in 39 patients on MRI and CT. Abnormal enhanced leptomeninges, indicating association with leptomeningitis, occurred in 35 cases. Secondary hydrocephalus existed in 10 cases. Moreover, cysticerci simultaneously infecting other sites outside the CNS, including orbital medial rectus muscle, the temporalis muscle and lingualis, were also found in 1 case.

\section{DISCUSSION}

Cysticercosis is a parasitic disease caused by larva of taenia solium, which parasitizes in human body as the intermediate host after ingesting of pork tapeworm eggs. Cysticercosis of the CNS (neurocysticercosis) is the most common parasitic infection in the CNS with predilection sites of meninges, parenchyma or ventricles (12). Neurocysticercosis is commonly seen in developing countries. Its incidence in China continues at a high level, especially in Southwest China. Due to the increase
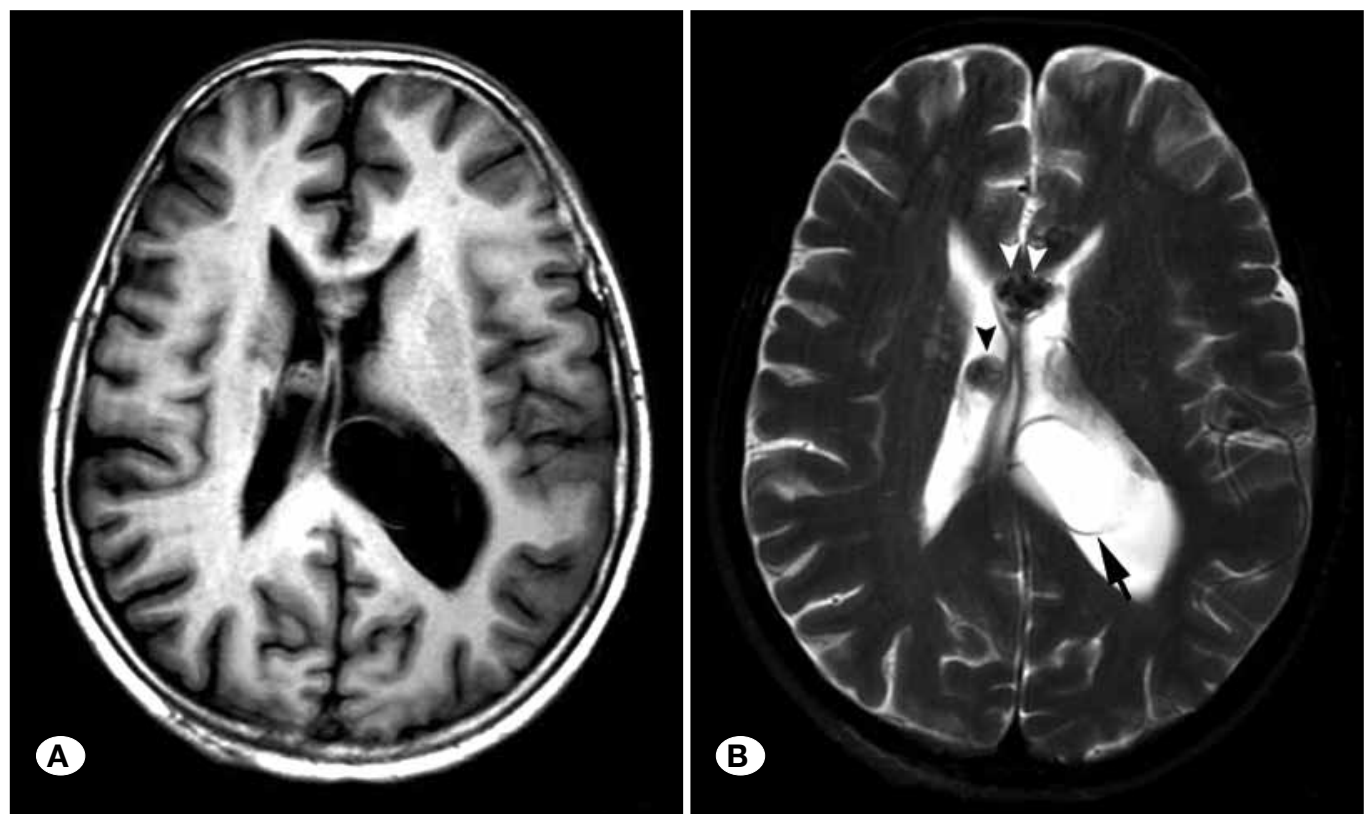

Figure 7: The MR imaging of a 40-year-old female with ventricular cysticercosis. A) T1WI, B) T2WI. A big cyst with a thin capsule as hypointensity, floating in the CSF of the left lateral ventricle, was displayed on T2WI (B. Arrow). Besides, the nodules with low signals in the right lateral ventricle and anterior septum pellucidum were seen on T1WI and T2WI (B. Arrowhead), indicating the calcified cysticerci.
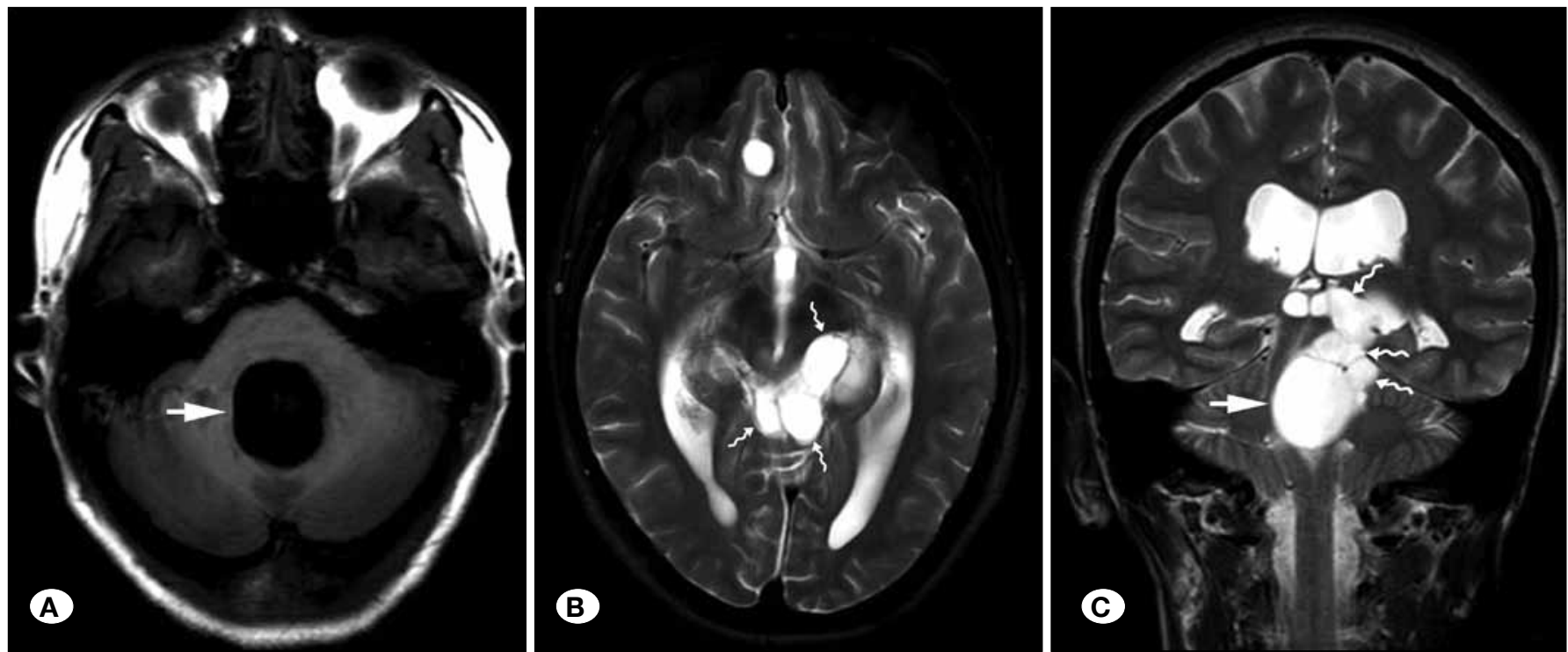

Figure 8: The MR imaging of a 31-year-old female with neurocysticercosis. A) T1WI, B) axial T2WI, C) coronal T2WI. The $4^{\text {th }}$ ventricle was filled by a huge cyst (A, C. Arrows), meanwhile, the left quadrigeminal cistern were involved by multiple different sized cysticerci with thin capsules (B, C. Curved Arrow). 
in immigration, the incidence in developed countries is also rising in recent years $(2,10)$. Clinical symptoms of neurocysticercosis are various, including seizure, headache, vomiting, limb movement disorder and disturbance of consciousness. Imaging examination is an important diagnostic means of neurocysticercosis, and it is therefore necessary to deepen the understanding of its imaging performance in order to reduce misdiagnosis rates. In 2001, experts formulated in detail the diagnosis of neurocysticercosis into 4 categories as absolute criteria, major criteria, minor criteria and epidemiologic criteria, based on the clinical manifestations, imaging findings, pathologic examinations, laboratory tests and epidemiological situations. According to the combination of these criteria, two levels of diagnostic certainty as definitive diagnosis and probable diagnosis were defined (4). In our study, 57 patients corresponding to definitive diagnosis were included.

According to the location in the CNS, neurocysticercosis can be divided into parenchymal cysticerci, subarachnoidal cysticerci, ventricular cysticerci and spinal cysticerci. In some publications, the subarachnoid space is the most common site, followed by the parenchyma (12). However, in our series, cysticerci located in the parenchyma were the most common with a percentage of $93 \%$, followed by subarachnoidal cysticerci with $68 \%$ and ventricular cysticerci with $23 \%$. Consistent with the literature, spinal cysticerci were the rarest, accounting for $2 \%$ only (Figure $9 \mathrm{~A}, \mathrm{~B}$ ).

Parenchymal cysticerci commonly locate in the corticaljuxtacortical junction, basal ganglia and cerebellum (12). On $\mathrm{MRI}$, the typical cysticercus is manifested as a target-like lesion, with a punctate nodule within a round vesicle. Due to limitations by the brain parenchyma, the cysts are generally smaller in size (3). In our series, parenchymal cysts smaller than $15 \mathrm{~mm}$ were found in 51 cases. With different phases of the neurocysticercosis, the imaging appearances of cysticerci vary in size, capsular thickness, perifocal edema and signal or density of the scolex. According to the imaging findings corresponding with the pathologic phases, Kimura-Hayama et al. classified neurocysticercosis into five stages as non-cystic stage, vesicular stage, colloidal-vesicular stage, granularnodular stage and calcified-nodular stage (8). In our series, cysts over $15 \mathrm{~mm}$ in diameter with thin capsules were seen in 8 cases. The scolexes of lesions had similar MR signals with brain parenchyma. On contrast-enhanced T1WI, the capsules and scolexes showed slight enhancement. Apart from mild edema seen in 4 cases, no perifocal edema was noticed on FLAIR and T2WI in the other 4 patients. These manifestations were consistent with the imaging features in the vesicular stage, due to the host immune tolerance and minimal inflammatory reaction at this stage. In addition, cysts smaller than $15 \mathrm{~mm}$, were displayed in 51 cases, with thick capsules, which were apparently enhanced on contrastenhanced T1WI. In 27 cases, the cysts, with mean diameter of $8 \mathrm{~mm}$, had serious edema around them. These manifestations corresponded to the imaging findings of cysticercosis with severe perifocal edema and incrassated capsules caused by inflammation after the death of larvae in the colloidal-vesicular stage. In 30 cases, the cysts with smaller size, mean diameter of $5 \mathrm{~mm}$, were shown as hypointensities with mild edema on T2WI, which were consistent with the imaging features in the granular-nodular stage with cystic shrinkage and inflammatory reduction. In 3 cases, multiple calcified lesions without obvious cystic cavity were displayed as punctate hyperdensities on CT, and extreme hypointensities on various sequences MRI. These were consistent with radiological features of the granulomatous cyst completely shrunk to a

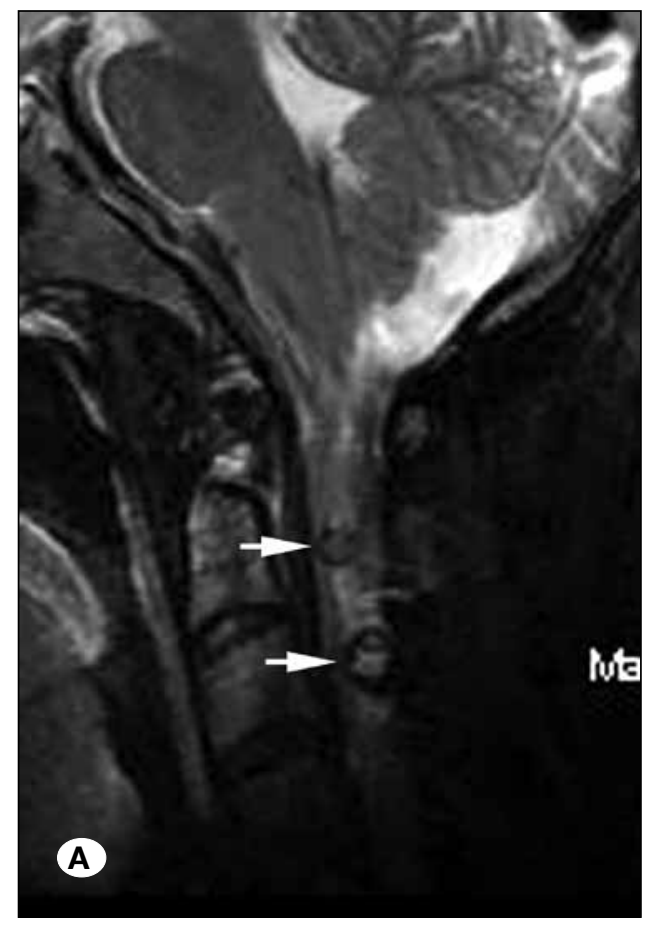

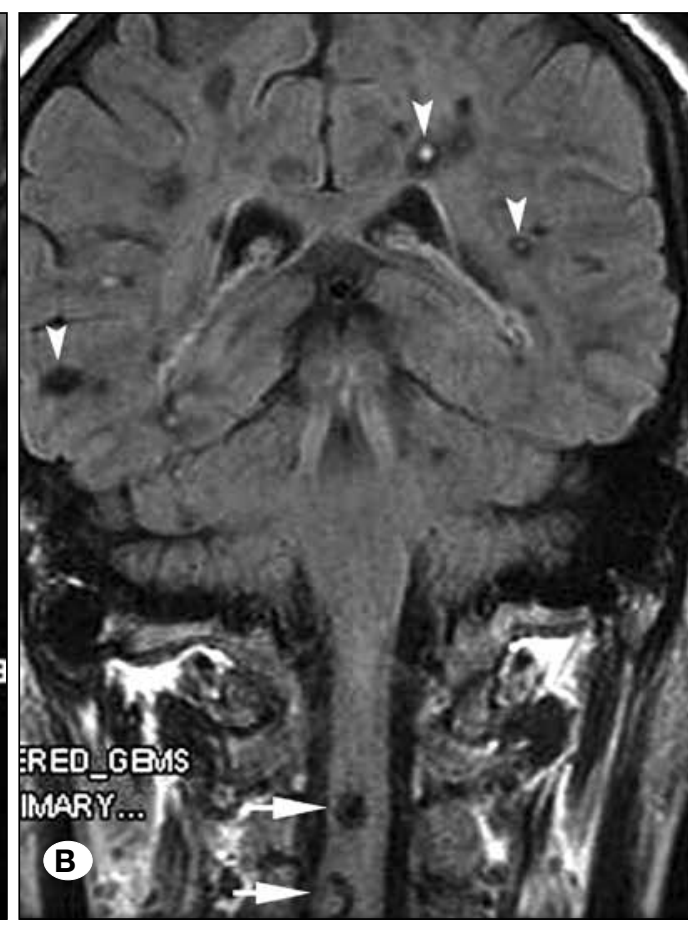

Figure 9: The MR imaging of a 46-year-old female with spinal cysticercosis. A) Sagittal T2WI, B) Coronal FLAIR. Two cyclic lesions located at the C1-2 level of the spinal cord appeared as peripheral hypointensity and central hyperintensity on T2WI (A, B; Arrow), with similar appearance of cavernous hemangioma. Moreover, multiple lesions with similar signals were found in bilateral parenchyma (B; Arrowhead). These low signal areas may indicate their stage of calcification. 
calcification nodule in the calcified-nodular stage. Besides, in 1 case, the cysticerci with multiple calcifications were similar to multiple cavernous hemangiomas on MRI. It is noteworthy that the above-mentioned various appearances could coexist simultaneously in the same patient, if the cysticerci are within different phases (7). Based on our imaging data, we believe that awareness of these imaging features has clinical value in determining the phase of the neurocysticercosis.

Subarachnoidal cysticerci are formed by larva of taenia solium infecting the subarachnoid space or the brain cistern via the blood circulation. In the literature, the subarachnoid cavity is the most common site infected by cysticerci (12). However, in our series, subarachnoidal cysticerci accounted for $68.4 \%$, less than the parenchymal cysticerci. The cysticerci of the cerebral convexity are commonly located in the sulci, with a small size due to the limitation by the surrounding tissues (12). The cysticerci in the Sylvian fissure or cisterns often have thin capsules and bigger sizes, and the maximum diameter reached $5 \mathrm{~cm}$ in our series. On MRI, the signals of cystic fluid are close to CSF signals with hypointensity on T1WI and hyperintensity on T2WI. Thus, sometimes, it is easy to ignore cisternal cysticerci on MR imaging. However, awareness of some signs such as the widened subarachnoid space, cystic signal difference between cystic fluid and CSF, and the adjacent leptomeningeal enhancement may help radiologists in the diagnosis. We also notice that, even on enhanced MRI, the scolexes were hardly displayed in cisternal cysticerci. According to the literature, this phenomenon is possibly formed by scolex degeneration and hydropic degeneration through continuous adsorption of cerebrospinal fluid to the vesicle $(3,7)$.

Ventricular cysticerci are relatively rare with incidence of $24.6 \%$ of our neurocysticercosis cases. The lesions are most commonly seen in the lateral ventricle and $4^{\text {th }}$ ventricle, but rarely found in the $3^{\text {rd }}$ ventricle $(1,8,11,12)$. The cysticerci located in the fourth ventricle are often big in size, and sometimes can occupy the whole $4^{\text {th }}$ ventricle. It often seems unsatisfactory to observe the cystic wall on the axial MRI scans only. Combination with sagittal and coronal imaging is helpful in observation of the outline of cysticercosis. As mentioned in the literatures, cysticerci in the ventricle could adhere to the ependyma or float freely in the ventricle (12). These findings were all found on the imaging in our series. The cysticercus with ependymal attachment commonly has a visible scolex and clear cystic wall, with ring-like enhancement on contrast-enhanced MRI. Meanwhile, some of intraventricular cysticerci had big vesicles, with thin cystic walls and few scolexes, and lack of enhancement on contrastenhanced T1WI. The cystic fluid signal was relatively higher on T1WI but lower on T2WI compared with the CSF signal. By noticing the signal differences between cystic fluid and CSF, and the occupying effect of the expanded ventricle, the clinicians and radiologists might get valuable information for the consideration of intraventricular cysticercosis.

Cysticercosis located in the spinal canal is very rare, less than $3 \%$ reported in the literature, and often occurs in the spinal subarachnoid space. Intramedullary cysticercosis is extremely rare $(<1 \%)$, and it is usually located in the dorsal spinal cord (9). On the image, the spinal cysticerci have similar manifestations with cerebral cysticerci. In our series, a rare case with intramedullary cysticerci in the calcified stage was found. Consistent with the literature, the lesions were located in the dorsal cervical spinal cord, with low signals on both $\mathrm{T} 1 \mathrm{WI}$ and $\mathrm{T} 2 \mathrm{WI}$ and an appearance similar to cavernous hemangioma, and the diagnosis was finally confirmed through the clinical and laboratory tests.

As for the differential diagnosis, parenchymal cysticerci should be differentiated from metastases, sarcoidosis, tuberculosis, and other parasites. While in the calcified stage, the lesions should be distinguished from the multiple cavernous hemangiomas or cerebral amyloid angiopathy on MRI. The differentiation of subarachnoid cysticerci includes arachnoid cysts, epidermoid cysts, dermoid cysts and tuberculous or fungal meningitis. The cysticerci in the spine should be differentiated from cystic astrocytoma, hemangioblastoma and ependymoma. The medical history, epidemiological situation and laboratory tests of the patient are crucial in the differential diagnosis. Besides, attention to the findings of the cystic wall and intracapsular scolex on MRI or CT is very important in identifying neurocysticercosis.

The treatment of neurocysticercosis is not fully defined yet, due to different numbers, locations and complications of the lesions $(2,6,8)$. In general, medical therapy is the first choice in consideration. Presently, albendazole and praziquantel are the most common and effective anti-cysticercosis drugs $(5,13)$. In the case of the symptoms with intractable seizures and acute severe intracranial hypertension, or the definite diagnosis of obstructive or communicating hydrocephalus resulting from intraventricular or subarachnoidal cysticerci, surgical therapy should be considered. In addition, for some undiagnosed isolated lesions with obvious mass effect, surgical resection is helpful not only in treatment but also in clarifying the diagnosis. In our 10 patients who underwent surgical treatment, 9 cases were diagnosed through the surgical resection while 1 case had a preoperative definite diagnosis.

\section{- CONCLUSION}

Neurocysticercosis is a common parasitic disease in developing countries, and is currently attracting attention in developed countries due to its increasing incidence $(2,10)$. The comprehensive diagnosis of neurocysticercosis should be combined with imaging findings, clinical history, epidemiologic data and laboratory examinations. The radiological methods have good value in displaying the extent and locations of the lesions. According to the numbers, cyst sizes, capsular thickness, densities or signals of the scolexes, as well as the perifocal edema, the developmental stages of neurocysticercosis could be determined in certain degrees. Therefore, these imaging features have a significant value in making possible timely diagnosis for clinicians, so as to arrange further examinations and guide proper treatments. 


\section{- ACKNOWLEDGEMENTS}

Gratitude has to be expressed to all the patients for participating in this research. Especially thank Dr. Xiao Jiahe for his support of acquisition of radiological data.

\section{- REFERENCES}

1. Cuetter AC, García-Bobadilla J, Guerra LG, Martínez FM, Kaim B: Neurocysticercosis: Focus on intraventricular disease. Clin Infect Dis 24(2):157-164, 1997

2. Del Brutto $\mathrm{OH}$ : Neurocisticercosis: Actualización en diagnóstico y tratamiento. Neurología 20:412-418, 2005 (In Spanish)

3. Del Brutto OH: Neurocisticercosis. Rev Neurol 29:456-466, 1999 (In Spanish)

4. Del Brutto OH, Rajshekhar V, White AC Jr, Tsang VC, Nash TE, Takayanagui OM, Schantz PM, Evans CA, Flisser A, Correa D, Botero D, Allan JC, Sarti E, Gonzalez AE, Gilman RH, García $\mathrm{HH}$ : Proposed diagnostic criteria for neurocysticercosis. Neurology 57:177-183, 2001

5. Del Brutto $\mathrm{OH}$ : Neurocysticercosis: A review. The Scientific World J 2012:159821, 2012

6. García HH, González AE, Tsang VCW, Gilman RH: Neurocysticercosis: Some of the essentials. Pract Neurol 6:288-297, 2006
7. Hawk MW, Shahlaie K, Kim KD, Theis JH: Neurocysticercosis: A review. Surg Neurol 63:123-132, 2005

8. Kimura-Hayama ET, Higuera JA, Corona-Cedillo R, ChávezMacías L, Perochena A, Quiroz-Rojas LY, Rodríguez-Carbajal J, Criales JL: Neurocysticercosis: Radiologic-pathologic correlation. Radiographics 30:1705-1719, 2010

9. Palacios E, Salgado Lujambio P, Rojas Jasso R: Computed tomography and magnetic resonance imaging of neurocysticercosis. Semin Roentgenol 32:325-334, 1997

10. Saavedra H, Gonzales I, Alvarado MA, Porras MA, Vargas V, Cjuno RA, Garcia HH, Martinez SM: Neurocysticercosis diagnosis and management in Peru. Rev Peru Med Exp Salud Pública 27:586-591, 2010

11. Sánchez L, Abad L, Lozano E, Maldonado G: Neurocisticercosis intraventricular. Presentación de un caso localizado en el tercer ventrículo. Radiología 44:309-313, 2002 (In Spanish)

12. Sarria Estrada S, Frascheri Verzelli L, Siurana Montilva S, Auger Acosta C, Rovira Cañellas A: Imaging findings in neurocysticercosis. Radiologia 55(2):130-141, 2013

13. Sotelo J, Diaz-Olavarrieta C: Neurocysticercosis: Changes after 25 years of medical therapy. Arch Med Res 41(1):62-63, 2010 